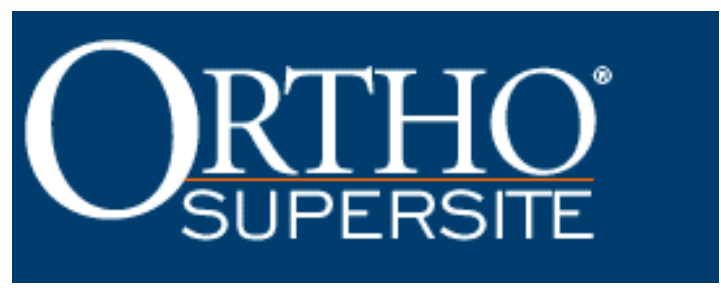

\title{
Painless Reduction of Acute Anterior Shoulder Dislocations Without Anesthesia
}

\author{
By Daniel R. O'Connor, MD, PC; Daniel Schwarze, MD; Austin T. Fragomen, MD; Marlene Perdomo, PA \\ ORTHOPEDICS 2006; 29:528
}

\section{June 2006}

\begin{abstract}
In 1938, Dr Henry Milch described a maneuver for the reduction of acute anterior shoulder dislocations consisting of shoulder abduction and external rotation with "pulsion" of the humeral head. Although many methods may be used to reduce the dislocated glenohumeral joint, the Milch technique is unique because of its gentle, effective, and relatively painless nature. This article studied the effectiveness of this technique on 76 consecutive acute anterior shoulder dislocations in 75 patients seen in our institution's two campuses over an 18-month period. Twelve shoulders had concomitant fractures of the greater tuberosity. All 76 shoulders were reduced on the first attempt. No anesthesia was used, and no complications were reported from the reduction maneuver. The Milch method is an ideal first line treatment for all acute anterior shoulder dislocations including those associated with fracture of the greater tuberosity.
\end{abstract}

Acute anterior dislocation of the glenohumeral joint is a common injury that the general orthopedist often treats throughout his/her career as many emergency department personnel and primary care providers are uncomfortable attempting reduction maneuvers. We have studied a reduction technique that is well tolerated, simple, safe, reliable, does not require anesthesia, can be performed without assistance, and can easily be taught to residents, physicians assistants, and emergency room personnel. This method for shoulder reduction helps ensure that patients are receiving efficient and compassionate treatment.

Henry Milch ${ }^{1}$ published the Treatment of Dislocation of the Shoulder in 1938 based on his experience with a technique introduced previously by Sir Astley Cooper. ${ }^{2}$ Milch performed his reductions by placing the arm in an fully abducted, externally rotated position without the use of traction. This was different from the more traditional reduction techniques that involved the use of traction counter-traction on the partially abducted or adducted shoulder. He felt that complete overhead abduction of the shoulder produced a position of "conical symmetry" between the muscles of the humerus, scapula, and thoracic wall (Figure 1). In this orientation, he believed the muscles that would otherwise prevent the reduction were co-liner allowing the humeral head to slide over the rim of the glenoid with a gentle "pulsion" or pushing maneuver. He argued that performing reduction maneuvers with the arm in the anatomic position was counterintuitive as the orientation of the many layers of muscle fibers that act across the shoulder joint was haphazard (Figure 2). He held that traction on the adducted upper extremity produced cross stresses in the shoulder stabilizing muscles requiring more force to achieve reduction. The increased force exerted would cause more pain, require the use of anesthesia, and increase the risk of associated complications. Milch performed his reductions without anesthesia or assistance.

Before undertaking this study, all acute anterior shoulder dislocations that presented to our institution were reduced using traction counter-traction techniques after administering conscious sedation. There were some disadvantages with this method. The use of conscious sedation led to delays in reduction, the need for multiple assistants and hospital personnel, exposure to potentially harmful medications, and delay in obtaining a post-reduction radiographs. Attempts at closed reduction without anesthesia

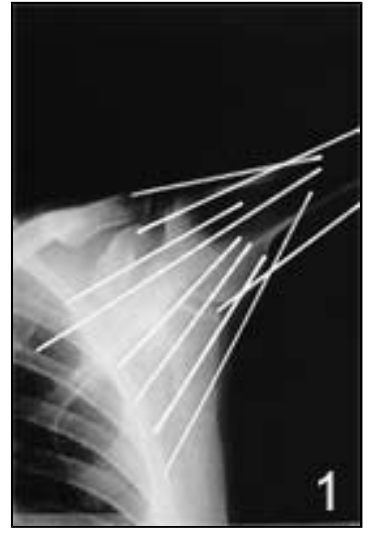

Figure 1: AP radiograph of the abducted externally rotated shoulder. White lines represent the vectors of the muscle forces acting across the glenohumeral joint. 
using traction methods were not well tolerated by patients. This study shows that the Milch shoulder reduction technique is an excellent alternative to the traditional methods. The Milch technique currently is the preferred technique at our institution.

\section{Materials and Methods}

Over an 18-month period, 75 consecutive patients with 76 acute anterior shoulder dislocations were treated in our institution's two emergency rooms either Mary Immaculate Hospital or Saint John's Queens Hospital both located in busy urban settings in Queens, New York. Patients initially were evaluated in the emergency department prior to orthopedic consultation. The emergency room physicians were made aware of this study and were instructed not to perform any reductions or administer any medications to participants. Clinical examination was performed and neurovascular status documented. Radiographs of the shoulder in the anteroposterior and lateral planes were obtained.

Inclusion criteria for the study included those patients with an acute anterior dislocation of the glenohumeral joint that was documented by radiographs to be displaced at the time of initial presentation. The dislocation must have occurred within 24 hours of the orthopedic consultation. Patients with dislocations that occurred $>24$ hours. Fracture dislocations other than those involving the greater tuberosity also were excluded. Patients who were pre-medicated in the emergency department were excluded. Voluntary dislocators, those without radiographically documented dislocations, and those patients with posterior dislocations were barred from the study. Previous stabilization surgery was not a criterion for exclusion; however, none of our patients had undergone operations on the affected shoulder. All of the 75 patients treated were included in the study as none met any of the exclusion criteria. There were $57(76 \%)$ men and $18(24 \%)$ women in the study. The mean patient age was 34 years. For 64 (91\%) patients, this injury represented an initial shoulder dislocation. The most common mechanism of injury for the first-time dislocators was a low-velocity fall. However, one patient sustained bilateral shoulder dislocations from a motorcycle accident. For those 11 patients with recurrent instability, raising the arm in bed and while showering were the predominant causes of the dislocation event. In all cases, radiographs confirmed an anteroinferior glenohumeral dislocation. A fracture of the greater tuberosity of the humerus was found in 12 shoulders. Seven of the 12 fractures were displaced $1-2 \mathrm{~cm}$, and the remaining five were displaced $<1 \mathrm{~cm}$. No other fracture pattern was observed. No patient in our series had sustained a neurological or vascular injury.
The conical symmetry of these lines indicates that the perihumeral musculature is in the optimal position to efficiently reduce the humeral head.

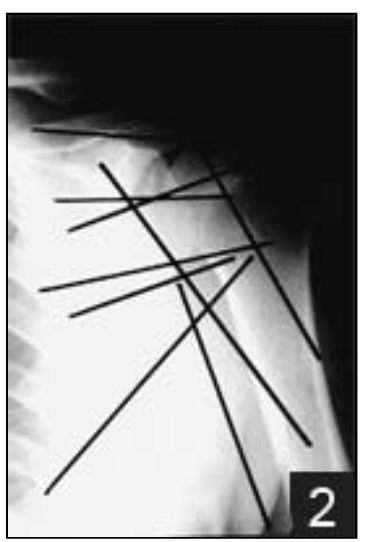

Figure 2: $A P$ radiograph of the shoulder in the anatomic position. Black lines indicate the vector of pull of the perihumeral muscles. The lines of action of these muscles take an oblique course and generate significant cross stresses when longitudinal traction is applied.

The primary authors (D.R.O. or D.S.) performed all emergency room consultations and shoulder reductions. No assistant was used. The Milch technique was used in every case to reduce the dislocated shoulder. Each patient was placed supine on the gurney and the head of the bed was elevated to between $20^{\circ}$ and $30^{\circ}$. The operator explained the maneuver to the patient. The affected arm was held by the wrist and was slowly abducted and externally rotated (Figure 3 ). The patient was constantly reassured that no pain would be felt. When resistance to motion was encountered, the physician would stop, wait for the patient to relax, and then continue. He would proceed in this way until the arm had reached approximately $90^{\circ}$ of abduction and $90^{\circ}$ of external rotation. It usually took between 5-10 minutes to reach this point. Most of the shoulders had spontaneously reduced having reached this position. If the head of the humerus had not reduced, a gentle amount of longitudinal traction was applied in line with the humerus. Milch did not implement traction during his reductions making our technique a modified version of the original description. The physician's free hand was placed into the axilla and used to exert lateral and superior pressure on the humeral head to complete the maneuver. The position of the humeral head was assessed clinically and radiographically after reduction. A repeat neurovascular examination then was performed. The patients then were placed in a shoulder immobilizer with the following recommendations: patients aged $<20$ years were immobilized for six weeks as the recurrence rate in this age group is high. ${ }^{3,4}$ The remaining patients were treated for three weeks with an immobilizer. The presence of a fracture of the greater tuberosity did not alter the treatment protocol. Follow-up was difficult to perform as the majority of patients quickly recovered from the trauma of the dislocation and did not return for further treatment.

\section{Results}

The Milch technique was successful in obtaining a shoulder reduction on the first attempt in 76 of 76 consecutive dislocated shoulders. Post reduction physical examination and radiographs confirmed an uncomplicated reduction in every case. No neurologic or vascular injury occurred as a result of the reduction. The 12 fractures of the greater tuberosity were noted to be reduced to within $\mathrm{a}<5 \mathrm{~mm}$ gap at the fracture site once the humeral head had been returned to its proper anatomic location. No new fractures were created in the reduction process. There was no 
palpable "clunk" accompanying reduction. In many instances, the only way that the operator knew that a reduction had been achieved was by the immediate relief of discomfort expressed by the patient. At times the patient was unaware that his shoulder had reduced. No patient received pain medication prior to the reduction maneuver. When comparing patients who dislocated for the first time with those who suffered from recurrent instability no difference was noted in difficulty of reduction. Both men and women equally tolerated the procedure well. Age did not affect the final outcome nor did the mechanism of injury impact the ease of reduction. None of the patients demanded the administration of narcotics or refused to continue the procedure at any point. Some patients with recurrent shoulder dislocations were dubious at the suggestion of shoulder reduction without anesthesia. Ultimately, they were impressed with the technique and with the relative brevity of the emergency department visit when compared with previous experiences.

\section{Discussion}

Reduction of the dislocated glenohumeral joint has been successfully achieved by various methods since ancient times.

Hippocrates described the oldest known method for shoulder reduction: grasping the ipsilateral wrist and applying longitudinal traction. Counter-traction was supplied by the operator's heel in the axilla. ${ }^{5}$ Variations of this technique were used throughout the middle ages using different fulcrums and at times excessive force.

Modern variations of these traction techniques are widely used today. Rockwood and Wirth ${ }^{6}$ advocate traction counter-traction as a preferred treatment method. Waldron and $\mathrm{Hazel}^{7}$ used body weight to apply traction. Most modern techniques are performed using analgesia and/or sedation and most require an assistant. The complications associated with different traction methods were categorized by Calvert et $\mathrm{al}^{8}$ in 1942 . They described a series of reductions involving the use of excessive traction forces that resulted in upper extremity dysfunction, amputations, and mortalities. The more severe iatrogenic injuries are of historical significance in modern surgery, nonetheless neurovascular compromise is known to result from contemporary traction techniques. ${ }^{6}$

Kocher ${ }^{9}$ published his results of a "new" technique in 1869 in Berliner klinische.His method involved maneuvering the arm through a series of rotational movements to lever the humeral head over the rim of the glenoid. His technique was unique in that it addressed the anatomic considerations of the dislocated shoulder. The Kocher technique required the generation of significant forces applied through the soft tissues at times resulting in a humeral shaft or neck fracture, avulsion of the rotator cuff, and injury to the vessels and nerves about the shoulder. ${ }^{10,11}$ Reports of these injuries have made many orthopedists hesitant to use this method. ${ }^{6}$

In 1900, Stimson ${ }^{12}$ reported his method of reducing shoulder dislocations. He recommended cutting a hole in the middle of a cot, placing the patient in the lateral position on the cot, and hanging the injured arm through that hole essentially placing the shoulder in $90^{\circ}$ of abduction. He then would hang weight from the wrist and wait for gravity to reduce the shoulder. Critics of this technique cite that it is time consuming and poorly tolerated by the patient. If sedatives are given then the prone position becomes an obstacle to monitoring the patient and protecting the airway. ${ }^{13}$

Milch's technique for shoulder reduction has been studied and reviewed in the literature. Janecki and Shahcheragh ${ }^{14}$ reported 50 consecutive reductions using the "forward elevation maneuver," as described by Cooper and Milch, in 1980. He administered acetaminophen with codeine at times, but no intravenous or intramuscular medications. He added gentle overhead traction and slight terminal internal rotation when necessary to complete the reduction. All shoulders were reduced on the first attempt with no complications. In 1989, Cortes et al ${ }^{13}$ reported his results of "reduction of acute anterior dislocations of the shoulder without anesthesia in the position of maximum muscular relaxation." He altered the Milch technique by pulling continuous significant traction on the shoulder during the maneuver. Although none of his patients received general anesthesia, two thirds were given analgesia and/or sedation. Of 128 patients studied, reduction was only achieved in $83 \%$ of cases. Russell et all ${ }^{15}$ reported on his experience with the Milch technique in injured skiers. In his series, 68 of 76 dislocations were reduced. His method involved instructing the patient to place their hand behind their head. The abducted, externally rotated position was achieved with little discomfort or assistance and in most cases without medication. Then gentle traction was applied, and the operator's free hand would guide the humeral head over the glenoid. Beattie et al ${ }^{10}$ conducted a prospective randomized study comparing the Milch and Kocher techniques for acute dislocations of the glenohumeral joint. He performed the Milch maneuver with the addition of gentle traction. He does not comment on his use of sedation. Seventy percent of the dislocations were successfully reduced on the first attempt using the Milch technique.

The variable results reported using the Milch maneuver are most likely of multiple etiologies. The use of strong traction is associated with increased pain and muscle spasm that may preclude shoulder reduction. In the series by Cortes et $\mathrm{al}^{13}$ series, increased muscle spasm and pain associated with traction necessitated the use of analgesia and sedation to perform the reduction. His success rate of $83 \%$ included those patients who were sedated at the 
time of attempted reduction. The $89 \%$ success rate of Russell et al, ${ }^{15}$ was superior to that of Cortes et al. ${ }^{13}$ Presumably, their use of gentler traction necessitated less analgesia and produced improved results. The use of gentle traction, although not advocated by Milch, had no negative affect on our results with $100 \%$ success rate on the first attempt. There is no clear explanation for the inferior results obtained by Beattie et al. ${ }^{10}$
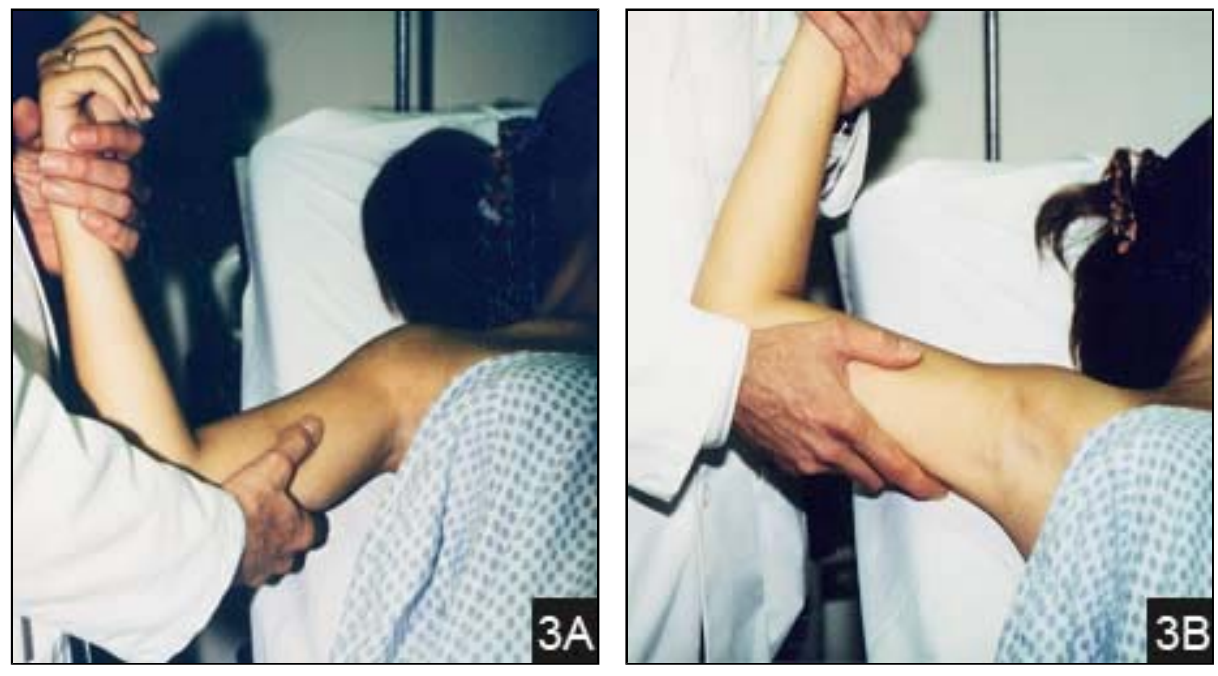

Figure 3: The shoulder is slowly abducted to $90^{\circ}(\mathrm{A})$ and externally rotated to approximately $90^{\circ}(\mathrm{B})$.

Although much time and effort has been dedicated to the study of the mechanics of shoulder dislocations, there is a scarcity of research describing how the mechanisms of popular shoulder reduction techniques address the pathoanatomy of the dislocated glenohumeral joint. The biomechanical explanation offered by Milch to prove the superiority of his technique over other contemporary methods of shoulder reduction is intuitive, but not supported by sound research. Milch ${ }^{1}$ maintained that the position of shoulder abduction and external rotation aligned the muscles of the shoulder girdle and eased the reduction when compared with other techniques. He opined that the haphazard arrangement of these muscles in the adducted shoulder prevented a gentle reduction. ${ }^{1}$ Basmajian and Bazant ${ }^{16}$ looked at the factors preventing the downward dislocation of the adducted shoulder. However, electromyography revealed that downward traction applied to the adducted shoulder resulted in minimal activity in the deltoid, biceps, and triceps muscles. However, they found that the supraspinatus tendon and the superior part of the capsule (including the coracohumeral ligament) were taut when subjected to downward loading and prevented inferior movement of the humeral head. They added that in the abducted position the head was easily subluxated as these superior structures were now lax. ${ }^{16}$ Based on these findings, one would expect that a subcoracoid dislocation of the humeral head would significantly increase the tension in the supraspinatus tendon and superior capsule. Longitudinal traction applied to the adducted dislocated shoulder should further increase stress applied to these superior structures making for a more painful experience and possibly increasing the risk of supraspinatus tendon damage.

The success of Milch's method for shoulder reduction most likely relies on the position of the capsulolabral structures more so than on the orientation of the muscles of the shoulder girdle. In the fully abducted-externally rotated position, the superior capsular structures and the supraspinatus tendon are lax. ${ }^{16,17}$ As the humeral head abducts and externally rotates during a dislocation event, the anteroinferior glenohumeral ligament tightens and then fails by bony fracture, labral avulsion, or attenuation. ${ }^{18}$ With the removal of the major restraint to anteroinferior displacement of the abducted-externally rotated humeral head, movement into and out of the joint will most easily be accomplished in this position. Adduction of the dislocated shoulder should increase tension in the supraspinatus tendon and the superior capsular structures making reduction more difficult. By repositioning the arm into its location at the time of injury, the intact ligaments should slacken and the humeral head should be permitted to glide back into the proper position.

The bony architecture of the glenohumeral joint may play a role in the ease of reduction as well. Pagnani et al ${ }^{19}$ described that the area of contact of the articular surface of the humeral head in the adducted position corresponded with the "bare area" of the glenoid. In the abducted shoulder, the articular contact becomes more peripheral on the glenoid implying a subtle loss of congruency from an increased head arc. The less congruent superior articular portion of the humeral head may allow it to slide more easily over the glenoid rim during reduction when the shoulder is in the in the abducted position. 
The presence of a Hill-Sachs fracture changes the shape of the humeral head as the head becomes hooked on the anteroinferior glenoid rim. Hovelius et $\mathrm{al}^{20}$ noted that Hill-Sachs lesions are created in $55 \%$ of primary anterior shoulder dislocations, and we have noted this lesion in nearly all traumatic shoulder dislocations that undergo arthroscopic stabilization. Hill-Sachs lesions that engage the anteroinferior glenoid may be more easily dislodged by abduction and external rotation of the humeral head.

Weaknesses of this study include the lack of a pain scale to more objectively quantify the patient's experiences with this reduction method and the lack of a proven anatomic mechanism behind the success of Milch's technique. Further research needs to be dedicated to studying the mechanics of reduction techniques and their effects on the pathoanatomy of the dislocated shoulder.

\section{Conclusion}

Our results using the Milch technique were excellent: all dislocations were reduced on the first attempt, there were no complications from the reduction, the technique was easy to perform, and potentially harmful medications were avoided. The process was relatively painless, only one operator was necessary, and there was excellent patient satisfaction. The Milch technique is ideal for the treatment of dislocations encountered in the field, in the office, and in busy hospital settings where help may be sparse. The Milch technique should be a first line treatment for all acute anterior shoulder dislocations and those fracture-dislocations only involving the greater tuberosity.

\section{What is already known on this topic}

- Anterior glenohumeral dislocation is one of the most common orthopedic injuries.

- Most reduction techniques require patient referral to the emergency department and the use of conscious sedation with monitoring.

- A reduction technique is needed that is rapid, painless, easy to learn, safe, and does not require analgesia or monitoring.

\section{What this article adds}

- This article supports the use of the Milch technique and has shown that it is an effective, painless, and efficient reduction method that often is overlooked.

- This study represents a large series of patients studied retrospectively and demonstrates superior results to previously published studies.

- The presence of a greater tuberosity fracture does not preclude the use of this technique.

\section{References}

1. Milch H. Treatment of dislocation of the shoulder. Surg Gynecol Obstet. 1938; 3:732-740.

2. Cooper BB, ed. A Treatise on Dislocations and on Fractures of the Joints. Am 2nd ed. Boston, Mass: Wait, Carter and Hendee, Boston Lilly; 1825.

3. Rowe CR. Prognosis in dislocations of the shoulder. J Bone Joint Surg Am. 1956; 38:957-977.

4. McLaughlin HL, Cavallaro WU. Primary anterior dislocation of the shoulder. Am J Surg. 1950; 80:615-621.

5. Brockbank W, Griffiths DL. Orthopaedic surgery in the sixteenth and seventeenth centuries. J Bone Joint Surg Br. 1948; 30:365-375.

6. Rockwood CA Jr, Wirth MA. Subluxations and dislocations about the glenohumeral joint. In: Rockwood CA Jr, Green DP, Bucholz RW, Heckman JD, eds. Fractures in Adults. Philadelphia, Pa: Lippincott Williams Wilkins; 1996:1249.

7. Waldron VD, Hazel D. Technique for reduction of shoulder dislocation. Orthop Rev. 1991; 10:563-566.

8. Calvert J, LeRoy M, Lacroix L. Shoulder dislocations and vascular injuries [in French]. J Chir. 1942; 58:337346.

9. Kocher, T. A new reduction method for shoulder dislocations [in German]. Berlin Klin Wochnschr. 1870; 7:101-106.

10. Beattie TF, Steedman DJ, McGowan A, Robertson CE. A comparison of the Milch and Kocher techniques for acute anterior dislocation of the shoulder. Injury. 1986; 17:349-352.

11. Connolly JF, ed. Management of Fractures and Dislocations: an Atlas. 2nd ed. Philadelphia, Pa: W.B. Saunders Co; 1981.

12. Stimson LA. An easy method of reducing dislocations of the shoulder and hip. Medical Record. 1900; 57:356-357.

13. Cortes VC, Garcia-Dihinx Checa L, Rodriguez Vela J. Reduction of acute anterior dislocations of the shoulder without anesthesia in the position of maximum muscular relaxation. Int Orthop. 1989; 13:259-262.

14. Janecki CJ, Shahcheragh $\mathrm{GH}$. The forward elevation maneuver for reduction of anterior dislocations of the 
shoulder. Clin Orthop. 1980; 164:177-180.

15. Russell JA, Holmes EM III, Keller DJ, Vargas JH III. Reduction of acute anterior shoulder dislocations using the Milch technique: a study of ski injuries. J Trauma. 1981; 21:802-804.

16. Basmajian JV, Bazant FJ. Factors preventing downward dislocation of the adducted shoulder joint. An electromyographic and morphological study. J Bone Joint Surg Am. 1959; 41:1182-1186.

17. Moseley HF, Overgaard B. The anterior capsular mechanism in recurrent anterior dislocation of the shoulder. Morphological and clinical studies with special reference to the glenoid labrum and the gleno-humeral ligaments. J Bone Joint Surg Br. 1962; 44;913-927.

18. Legan JM, Burkhard TK, Goff WB II, et al. Tears of the glenoid labrum: MR imaging of 88 arthroscopically confirmed cases. Radiology. 1991;179:241-246.

19. Pagnani MJ, Galinat BJ, Warren RF. Glenohumeral instability. In: DeLee JC, Drez D, Miller MD, eds. Delee and Drez's Orthopaedic Sports Medicine: Principles and Practice. Vol 1. Philadelphia, Pa: Elsevier; 1994:580-622.

20. Hovelius L, Erikkson K, Fredin $\mathrm{H}$, et al. Recurrences after initial dislocation of the shoulder: results of a prospective study of treatment. J Bone Joint Surg Am. 1983; 65:343-349.

\section{Authors}

Dr O'Connor and Ms Perdomo are from the St Vincent Catholic Medical Centers, St John's Queens Hospital, Elmhurst, NY; Dr Schwarze is from St Louis, Mo; and Dr Fragomen is from the Hospital for Special Surgery, New York, NY.

Reprint requests: Austin T. Fragomen, MD, Hospital for Special Surgery Institute for Limb Lengthening and Reconstruction, 519 E 72nd St, Ste 204, New York, NY 10021.

\section{Copyright $\circledR_{2005}$ SLACK Incorporated. All rights reserved.}

